Mon. Not. R. Astron. Soc. 000, 000-000 (0000) Printed 27 April $2022 \quad$ (MN IATEX style file v2.2)

\title{
Escape of High Energy Particles from Bow-Shock Pulsar Wind Nebulae
}

\author{
N. Bucciantini ${ }^{1,2,3 \star}$ \\ ${ }^{1}$ INAF - Osservatorio Astrofisico di Arcetri, Largo E. Fermi 5, I-50125 Firenze, Italy \\ ${ }^{2}$ Dipartimento di Fisica e Astronomia, Università degli Studi di Firenze, Via G. Sansone 1, I-50019 Sesto F. no (Firenze), Italy \\ ${ }^{3}$ INFN - Sezione di Firenze, Via G. Sansone 1, I-50019 Sesto F. no (Firenze), Italy
}

Accepted / Received

\begin{abstract}
The detection of bright X-ray features and large $\mathrm{TeV}$ halos around old pulsars that have escaped their parent Supernova Remnants and are interacting directly with the ISM, suggest that high energy particles, more likely high energy pairs, can escape from these systems, and that this escape if far more complex than a simple diffusive model can predict. Here we present for the first time a detailed analysis of how high energy particles escape from the head of the bow shock. In particular we focus our attention on the role of the magnetic field geometry, and the inclination of the pulsar spin axis with respect to the direction of the pulsar kick velocity. We show that asymmetries in the escape pattern of charged particles are common, and they are strongly energy dependent. More interestingly we show that the flow of particles from bow-shock pulsar wind nebulae is likely to be charge separated, which might have profound consequences on the way such flow interacts with the ISM magnetic field, driving local turbulence.
\end{abstract}

Key words: MHD - ISM: cosmic rays - ISM: supernova remnants - magnetic fields - stars: pulsars: general

\section{INTRODUCTION}

Once escaped from their parent Supernova Remnant (SNR), usually a few tens of thousands of years after the Supernova explosion, pulsars (PSRs) begin to interact directly with the ISM (Gaensler \& Slane 2006, Bucciantini \& Bandiera 2001). The ram pressure balance between the pulsar wind and the supersonic ISM flow (in the reference frame of the PSR itself) leads to the formation of a cometary nebula known as bow-shock pulsar wind nebula (BSPWN) (Wilkin 1996, Bucciantini \& Bandiera 2001, Bucciantini 2002). These nebulae have been observed in $\mathrm{H}_{\alpha}$ emission, due to neutral Hydrogen of the ISM interacting through chargeexchange with the ionized component of the ISM itself, shocked in the outer bow-shock (Kulkarni \& Hester 1988, Cordes, Romani \& Lundgren 1993; Bell et al. 1995; van Kerkwijk \& Kulkarni 2001, Jones, Stappers \& Gaensler 2002; Brownsberger \& Romani 2014 Romani, Slane \& Green 2017), and in non-thermal radio/X-rays associated with the shocked pulsar wind, flowing backward into long tails (Arzoumanian et al. 2004, Kargaltsev et al. 2017| 2008, Gaensler et al. 2004, Yusef-Zadeh \& Gaensler 2005; Li, Lu \& Li 2005, Gaensler 2005, Chatterjee et al. 2005, Ng et al. 2009, Hales et al. 2009, Ng et al. 2010, De Luca et al.|2011, Marelli et al.|2013, Jakobsen et al. 2014, Misanovic, Pavlov \& Garmire 2008, Posselt et al. 2017, Klingler et al. 2016, Ng et al. 2012).

\footnotetext{
^ E-mail: niccolo@arcetri.astro.it
}

Modeling of BSPWNe have progressed in the last decades from simple classical axisymmetric hydrodynamics (Bucciantini 2002) to relativistic MHD (Bucciantini, Amato \& Del Zanna 2005) and full and/or simplified 3D (Vigelius et al. 2007; Bucciantini 2018, Barkov \& Lyutikov 2018). All of those models are based on the assumption that the pulsar wind behaves as a fluid, and as such is fully confined within a contact discontinuity (CD) bounding the non-thermal tail. However, old pulsars are often found embedded into extended TeV halos (Gallant 2007, Helfand et al. 2007, H. E. S. S. Collaboration et al. 2014 2018; Abeysekara et al.2017), that do not resemble at all the cometary structures found in simulations. It has been suggested that those PSRs might be slowly moving and still confined into their parent SNR. However more recently an extended $\mathrm{TeV}$ halo was found surrounding Geminga (Abeysekara et al. 2017), which is a well known BSPWN (Posselt et al. 2017), where X-rays clearly show a cometary shape. On the other hand, in the Guitar nebula associated with PSR B2224+65 (Hui \& Becker 2007) and in the Lighthouse nebula associated to PSR J1101-6103 (Pavan et al.2014), bright X-ray features clearly protruding out of the system, almost orthogonal to the PSR motion, are observed, that contrast strongly with what fluid models predict.

Previous studies have attempted to explain the presence of one-sided X-ray features invoking a preferential collimated escape of high energy particles from the PWN (Bandiera 2008). Such directed outflows have been associated with localized reconnection at the magnetopause (the $\mathrm{CD}$ ) between the magnetic field line of 
the PWN, and those of the ISM, in a fashion not dissimilar to what is known to happen on the dayside Earth magnetopause, where it interacts with the solar wind (Scholer 2003; Frey et al. 2003, Faganello et al.2012, Fuselier et al. 2012 2014). However reconnection at the magnetopause is known to be patchy and sporadic and to lead only to marginal flux transfer (Kan 1988, Pinnock et al. 1995. Fear et al. 2017) in contrast with the persistence of those X-ray features, and on the large energetics (comparable to the PSR spindown) required to power them. Moreover, reconnection is likely to affect only those low energy particles that are bound to follow magnetic field lines, while existing models invoke the presence of particles with typical Larmor radii comparable with the size of the bow-shock, that are likely unable to feel the existence of small reconnecting regions.

The problem of the escape of particles from old BSPWNe, is also relevant in the context of dark matter searches. Pulsars are likely the most efficient antimatter factories in the Galaxy, and BSPWNe have could be one of the major, if not the main, contributor to the positron excess observed by PAMELA (Adriani et al. 2009. Hooper, Blasi \& Dario Serpico 2009, Blasi \& Amato 2011. Adriani et al. 2013, Aguilar et al. 2013), in competition with dark matter (Wang, Pun \& Cheng 2006).

Of course the flow dynamics in these systems can be quite complex, and many key factors (turbulence, differential acceleration at the termination shock, etc...) can lead to anisotropies in the escape of high energy particles. Here, however, our interest is focused in singling out the specific role of the magnetic field geometry, using a simplified model for the magnetic field structure in the head of these nebulae. We trace the trajectories of charged particles that emerge out of the PWN in its head, and assess the level of anisotropy associated to the emerging flow.

In Sect. 2 we describe how we model the magnetic field structure and particle trajectories. In Sect. 3 we illustrate our results for different magnetic field configurations and particle rigidities. In Sect. 4 we state our conclusions.

\section{MODELING PARTICLES TRAJECTORIES}

We are interested here in understanding the level of anisotropy that the magnetic field can induce in the escape of high energy particles from the head of a typical BSPWN. Given that only particles with typical energies close to the pulsar voltage are expected to escape efficiently from the head of these nebulae (Bandiera 2008), and that for these particles, the Larmor radius in the typical ISM magnetic field is much larger than the size of the bow shock, only the PSR wind magnetic field compressed in the head of these nebulae can in principle introduce anisotropies in their escape. For this reason we only model the magnetic field inside the bow-shock CD.

The magnetic field structure and the flow geometry are modelled according to the recipe described in Bucciantini (2018), to which the reader is referred for a detailed discussion. Here we remind briefly their properties. The flow speed is assumed to be purely laminar, and time independent. Within the PSR wind termination shock (TS) the flow is purely radial and moves at the speed of light $c$. In the region bounded by the TS, on the inner side, and the $\mathrm{CD}$, on the outer side, we use a semi-analytical prescription, that approximates the average flow conditions, as found in $2 \mathrm{D} \mathrm{nu}-$ merical simulations Bucciantini 2002, Bucciantini, Amato \& Del Zanna 2005). The flow is assumed to be purely axisymmetric, with the symmetry axis given by the direction defined by the PSR kick velocity with respect to the ISM. On top of this flow we compute the magnetic field structure, evolving the induction equation for a passive solenoidal field in the steady state regime, assuming that in the PSR wind the field is purely toroidal. This is done for various inclinations $\theta_{\mathrm{i}}$ [see Fig. 3 of Bucciantini (2018)] between the PSR spin axis and the PSR velocity, and for different dependencies of the magnetic field strength on the polar angle $\Psi$ with respect to the PSR spin axis itself. In the PSR wind, whence the field is injected, the value of the magnetic field is:

$$
B=B_{o}\left(\frac{d_{o}}{2 r}\right) \times\left\{\begin{array}{l}
1-2 \Theta[\pi / 2-\Psi] \quad \text { Case A } \\
\sin (\Psi)(1-2 \Theta[\pi / 2-\Psi]) \quad \text { Case B } \\
\sin (\Psi) \tanh (\pi / 2-\Psi) \quad \text { Case C }
\end{array}\right.
$$

where $r$ is the distance from the PSR (which we locate at the center of our coordinate system), $\Theta$ is the Heaviside function that ensures the correct reversal of magnetic polarity at the PSR wind equator, and $d_{o}$ the bow-shock stand-off distance (the distance from the PSR of the $\mathrm{CD}$ in the head of the bow-shock) defined as:

$$
d_{o}=\sqrt{\frac{L}{4 \pi c \rho_{o} V^{2}}}
$$

where $L$ is the pulsar spin-down luminosity, $\rho_{o}$ is the ISM density (the density of the ionized component), and $V$ is the relative speed of the PSR with respect to the ISM. $B_{o}$ is the reference strength of the wind magnetic field at a distance from the PSR equal to half the stand-off distance. Case A represents a field with uniform strength, and a uniform magnetic energy flux in the wind. Case B instead corresponds to a magnetic energy flux that scales as $\sin ^{2}(\Psi)$ a prescription often adopted in PWNe numerical simulations (Komissarov \& Lyubarsky 2004, Del Zanna, Amato \& Bucciantini 2004, Bogovalov et al. 2005: Volpi et al. 2008, Porth, Komissarov \& Keppens 2014, Olmi et al. 2016, Del Zanna \& Olmi 2017) and based on force free models of the pulsar magnetospheres (Contopoulos, Kazanas \& Fendt 1999, Timokhin 2006, Spitkovsky 2006). Case $\mathrm{C}$ adds a further modulation that suppresses the magnetization in a large sector around the pulsar equator, and corresponds to the case of a striped wind emanating from an oblique rotator, where the magnetic field of the stripes is dissipated (Lyubarsky \& Kirk 2001, Kirk \& Skjæraasen 2003, Lyubarsky 2003, Komissarov \& Lyubarsky 2004, Del Zanna, Amato \& Bucciantini 2004).

On top of the magnetic field structure found in the various cases, and for various inclinations $\theta_{\mathrm{i}}$, we compute the trajectories of high energy charged particles, integrating the equations for energy and momentum, under the action of the total Lorentz force, where the electric field is given by the Ideal MHD condition, the flow speed and the magnetic field. Given that the flow structure and the magnetic field geometry, in our simplified approach, are independent of the magnetic field strength (the magnetic field is passive), the trajectories of the particles are just a function of the effective rigidity which we parametrize as:

$$
\mathcal{R}=\frac{m c^{2} \gamma_{i}}{q B_{o}} \frac{1}{d_{o}}=\frac{R_{\mathrm{lo}}}{d_{o}}
$$

where $m$ is the mass of the particle, $q$ the modulus of its charge, $\gamma_{i}$ its Lorentz factor at injection. $\mathcal{R}$ is just the ratio of the Larmor radius that the particle has in a field of strength $B_{o}$, with respect to the stand-off distance. Particles for which $\mathcal{R} \ll 1$ are strongly tied to the flow and unlikely to efficiently escape, while particles for which $\mathcal{R} \gg 1$ escape the bow-shock unaffected by the magnetic field. Particles with $\mathcal{R}=1$ have typical Larmor radii, in the typical magnetic field of the head, comparable to the stand-off distance. It can be shown (Bandiera 2008) that the typical energies of these particles 
ranges from about $10 \mathrm{TeV}$, for a pulsar like PSR B2224+65 in the Guitar nebula, to a few hundreds $\mathrm{TeV}$, for a pulsar like Geminga, PSR J0633+1746.

Given that we are interested in the possible escape from the very head of these nebulae, particles are followed in their trajectories until they either reach the $\mathrm{CD}$, or the move backward at a distance exceeding $2 d_{o}$ from the PSR, at which point we consider them lost in the tail. It is still likely that those particles can escape from the tail, but given that the tail might be in general more turbulent than the head we expect the escape there to be more isotropic.

In principle, the canonical model of PWNe assumes that particles are accelerated at the wind TS. However, for simplicity, given that we are interested in evaluating the level of anisotropy introduced by the magnetic field, we assume all high energy particles to be injected from the PSR (or equivalently the pulsar wind), with an isotropic distribution, and in the radial direction.

\section{RESULTS}

Using the magnetic structure computed according to the recipes of Bucciantini 2018) we injected $10^{5}$ particles with high Lorentz factor $(v \rightarrow c)$, isotropically from the pulsar, and we followed their trajectories until they reached the $\mathrm{CD}$. This was done for both signs of charge. Note that the trajectories of particles of the same energy depend on the sign of their charge with respect to the magnetic field polarity, which in turn is related to the inclination angle between the pulsar dipole moment and the pulsar spin axis. A trajectory of an electron in a toroidal magnetic field left-handed with respect to the spin axis, is the same as the trajectory of a positron of the same energy, in a right-handed field. Given that there is no way of measuring the polarity of the magnetic field, one cannot attribute a specific pattern of trajectories to either positrons/protons or electrons.

Our model allows us to locate the escape position along the $\mathrm{CD}$, to count the fraction of particles that escape in the head, and also to evaluate the energy gains/losses they experience as they move within the BSPWN.

We will focus our discussion on three kind of anisotropies:

- head-to-tail anisotropy: due to the fact that the magnetic field compression, the flow speed and the thickness of the BSPWN between the TS and CD varies substantially between the head and the tail;

- inclination anisotropy: due to the different geometry of the magnetic field, and the associated current layers, for different inclinations of the PSR spin axis with respect to the PSR kick velocity;

- charge anisotropy: due to the fact that particles of different sign behave differently on current layers/lines, in terms of confinement and collimation.

\subsection{Fully Axisymmetric Case}

If $\theta_{\mathrm{i}}=0^{\circ}$ the magnetic field structure is fully axisymmetric, and the magnetic field if fully azimuthal. In this case, the induction electric field is also purely poloidal, and the same holds for the total Lorentz force. The trajectories of the particles are bound to be confined in meridional planes, at fixed azimuthal angle. This implies that there is no inclination anisotropy. In Fig. 1 we show the trajectories of particles injected at different polar angles $\Psi$ along a meridional cut of the BSPWN, for $\mathcal{R}=0.25$ and different sign of the charge. It is evident that in this regime the trajectories are strongly dominated by the flow advection, with gyration playing only a minor role. It

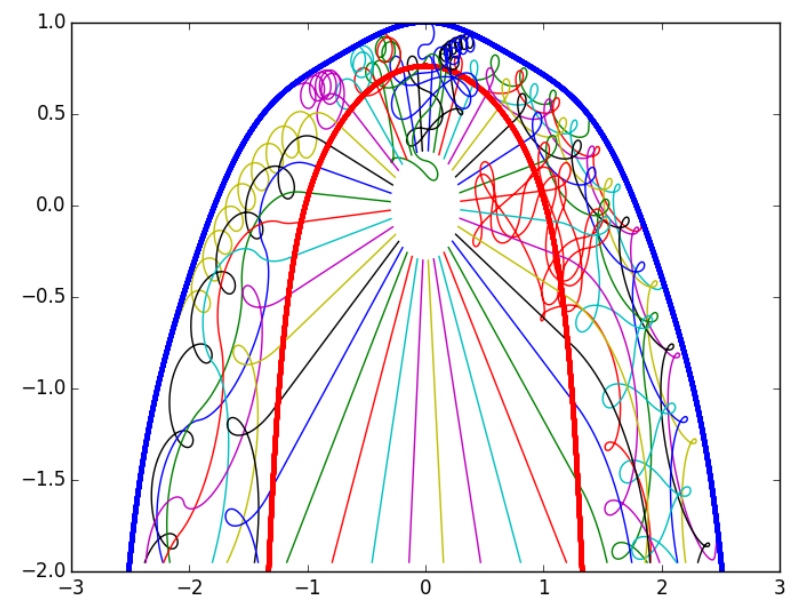

Figure 1. Trajectories of charges particles in the head of a BSPWN, for $\mathcal{R}=0.25$. Left part (negative abscissae) and right part (positive abscissae) represent trajectories of particles of different signs. The thick red curve is the PSR wind TS, while the thick blue curve is the CD. Axes are in units of the stand off distance (the size of the CD in the head).

is interesting to note that along the axis, in the head of the nebula, in the region corresponding to the location of the polar current line (the symmetry axis of the toroidal magnetic field), while particles of one sign tend to be confined, the particles of the opposite sign tend to be expelled. This is a common property of current lines/sheets, where magnetic field reverses sign. Moreover one can see in the tail the change in the direction of gyration, associated with the change of the magnetic field polarity due to the reversal of the field in the equatorial plane of the PSR rotation. It is evident that a large fraction of particles are advected in the tail. and only a small fraction manages to reach the CD in the head. In Fig. 2 we illustrate schematically how charged particles behave in the presence of current lines/sheets. At least as long as the the magnetic field preserves its azimuthal geometry, the polar current lines will confine particles of one sign, while the equatorial current sheet will confine particles of the opposite sign. Of course this is an energy dependent statement. In the limit $\mathcal{R} \rightarrow 0$ particles are tied to the flow, while for $\mathcal{R} \rightarrow \infty$ particles will not feel the magnetic field. In general we found that for $\mathcal{R}=3$ the location of the particle escape points on the $\mathrm{CD}$ is almost isotropic, and the totality of particles that are injected escapes. On the other hand for $\mathcal{R}<0.1$ the fraction of particles that escape in the head drops below $5-10 \%$, and the vast majority is advected in the tail.

In Fig. 3 we show the exit points of charged particles in polar coordinates along the $\mathrm{CD}$, for various values of the effective rigidity $\mathcal{R}$ and for different latitudinal shapes of the magnetic field. The appearance of one or more rings is due to the combination of gyration and advection, that leads to a periodic pattern. In Case A for small vales of $\mathcal{R}$ one sees that the escape points, apart from a concentration/depletion in the very head, due to the presence of the polar current line, tend to be uniformly distributed, compatible with the picture of an advection dominated motion. For higher values of $\mathcal{R}$, once the typical gyration radius becomes comparable with the size of the system, one begins to see large modulations in the escape pattern, with regions devoid of escaping particles. In Case B, one finds that, due to the sin-like modulation of the magnetic field in the head, the pattern in the head is changed. For $\mathcal{R}=1$ one begins 


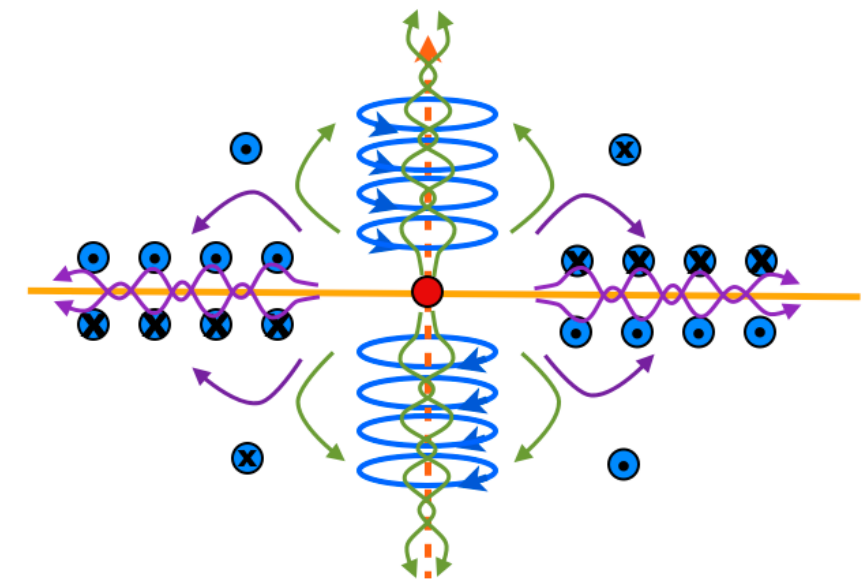

Figure 2. Schematic representation of the trajectories of high energy particles in the azimuthal magnetic field, injected by the PSR wind (red sphere). The magnetic field, with its polarity is shown in blue. The dashed orange line is the polar current line, while the orange solid line represents the equatorial current sheet. The trajectories of charges of different signs are shown in green and purple.

to see a pattern close to the free escape limit. In Case $\mathrm{C}$, where a large low-magnetization equatorial outflow tends to lower the mean magnetic field in the head, patterns much closer to the free escape limit are already reached at $\mathcal{R}=0.5$. Indeed we find that the escape fraction for $\mathcal{R}=0.5$ approaches $50 \%$ of the particle injected, and values close to $100 \%$ for $\mathcal{R}=1$. Interestingly for $\mathcal{R}<1$ the escape fraction of particles of different sign can differ by a factor up to 2. On the other hand, the energy gains/losses are only marginal, at most a factor of 2. It is evident, from the large diversity of patterns, that there is a strong dependence on the energy of the injected particles, on the specific shape of the CD and TS. However, even if the pattern might be sensitive to these specific choices of configuration, it is clear that for $\mathcal{R}<0.5$ large head-to-tail and charge anisotropies might be present, with charges of different signs escaping at different locations along the BSPWN.

\subsection{The Orthogonal Case}

In the orthogonal case, $\theta_{\mathrm{i}}=90^{\circ}$, it is the equatorial current sheet that extends all the way to the CD of the BSPWN, while the polar current lines remain confined inside the nebula. In this case one can clearly observe, as shown in Fig. 4, the charge anisotropy due to the presence of the equatorial current sheet. While particles of one sign tend to be strongly concentrated in the current sheet, those of the opposite sign are strongly defocused. In this case the number of escaping particles of opposite signs can differ even by a factor 2-3. In cases $\mathrm{A}$ and $\mathrm{B}$, for $\mathcal{R}<0.5$ less than $10 \%$ of the particles manage to reach the $\mathrm{CD}$, away from the curent sheet. For $\mathcal{R}=1$, in case $\mathrm{B}$, where the magnetic field close to the polar line is suppressed in a sin-like fashion, one begins to see the escape of particles confined in the polar region. In case $\mathrm{C}$, where the equatorial current layer is broad, we clearly see for $\mathcal{R} \geqslant 0.5$ a pattern that rapidly approaches the isotropic case, even if a marginal residual asymmetry between the escape points of the two charges (one more concentrated in the equatorial plane, one more along the polar axis direction) remains. Interestingly the escape probability for $\mathcal{R}<1$ in the orthogonal case if smaller than in the aligned case, suggesting that the geometry of the field provides a better confinement. More interesting is the fact that the fraction of escaping particles of different sign, at the same $\mathcal{R}$ can differ even by a factor a few. This mostly happens for small $\mathcal{R}<0.5$, and is due to the confinement effect of the current sheet. Indeed the excess is almost completely due to particle escaping along the equatorial current sheet. As before, the energy gains/losses are comparable and not exceeding a factor a few.

\subsection{Inclined Case}

In the inclined case $\theta_{\mathrm{i}}=45^{\circ}$ we see, as shown in Fig. 5, the same pattern due to the charge confinement in the equatorial current sheet, which translates into a clear asymmetry between the upper and lower part of the CD. Again we find a marked difference in the number of escaping particles between the two different charges, with escape fraction smaller than a few percent for $\mathcal{R}<0.25$. The presence of the equatorial current sheet, which now extends to the bottom part of the $\mathrm{CD}$, and of a polar current line in the upper part becomes evident at $\mathcal{R}>0.5$ in the escape pattern of the opposite charges. This top-bottom asymmetry is still present for $\mathcal{R}=1$ even in case $\mathrm{C}$ where both the polar current line and equatorial current sheet are broader. Similar to the previous cases there are regions on the $\mathrm{CD}$ where no escaping particle is present. Even in this case, gain and losses are in general negligible. As in the orthogonal case, even here we do observe a marked difference in the number of escaping particles of different signs.

\section{CONCLUSIONS}

Using a simplified model for the flow geometry and magnetic field structure in the head of a bow-shock pulsar wind nebula, we have investigated the escape of high energy charged particles, and in particular the role of the magnetic field in creating anisotropies in the escaping flow. Following their trajectories in the electric and magnetic field, we have try to assess how much the escape probability depends on the magnetic field geometry, in terms of strength of the field (the particle effective rigidity), the relative inclination of the pulsar spin axis and pulsar kick velocity, and in particular we focus on various kinds of anisotropies: head to tail, inclination, and especially charge anisotropy.

We want to stress here that there are several other possible sources of asymmetry in the escape of particles from BSPWNe: the energy flow in the wind can have a strong latitudinal dependence (Spitkovsky 2006, Tchekhovskoy, Philippov \& Spitkovsky 2016) which will affect the shape of the termination shock, and the structure of the flow downstream of it, leading to the formation of fast channels (Del Zanna, Amato \& Bucciantini 2004); particles acceleration at the shock can depend strongly on the local conditions, in term of inclination and magnetization (Spitkovsky 2008, Sironi \& Spitkovsky 2009, 2011, such that the shock itself could introduce major asymmetries in the way particle are in injected; shear at the $\mathrm{CD}$ between the internal pulsar wind flow moving at a speed $\sim 0.3-0.6 c$, and the slow moving outer shocked ISM (with velocity comparable to the pulsar kick velocity), can produce KelvinHelmholtz instability which might disrupt the magnetopause that is found at the CD in laminar models, and introduce a further source of scattering and turbulence inside the nebula. This will likely tend to isotropize the escape.

All of these effects can alter substantially the resulting escape pattern, and they can also introduce major time dependencies. However, even if the pattern of escape particles can differ substantially from what we found, there are a few results from our study that we deem robust: 

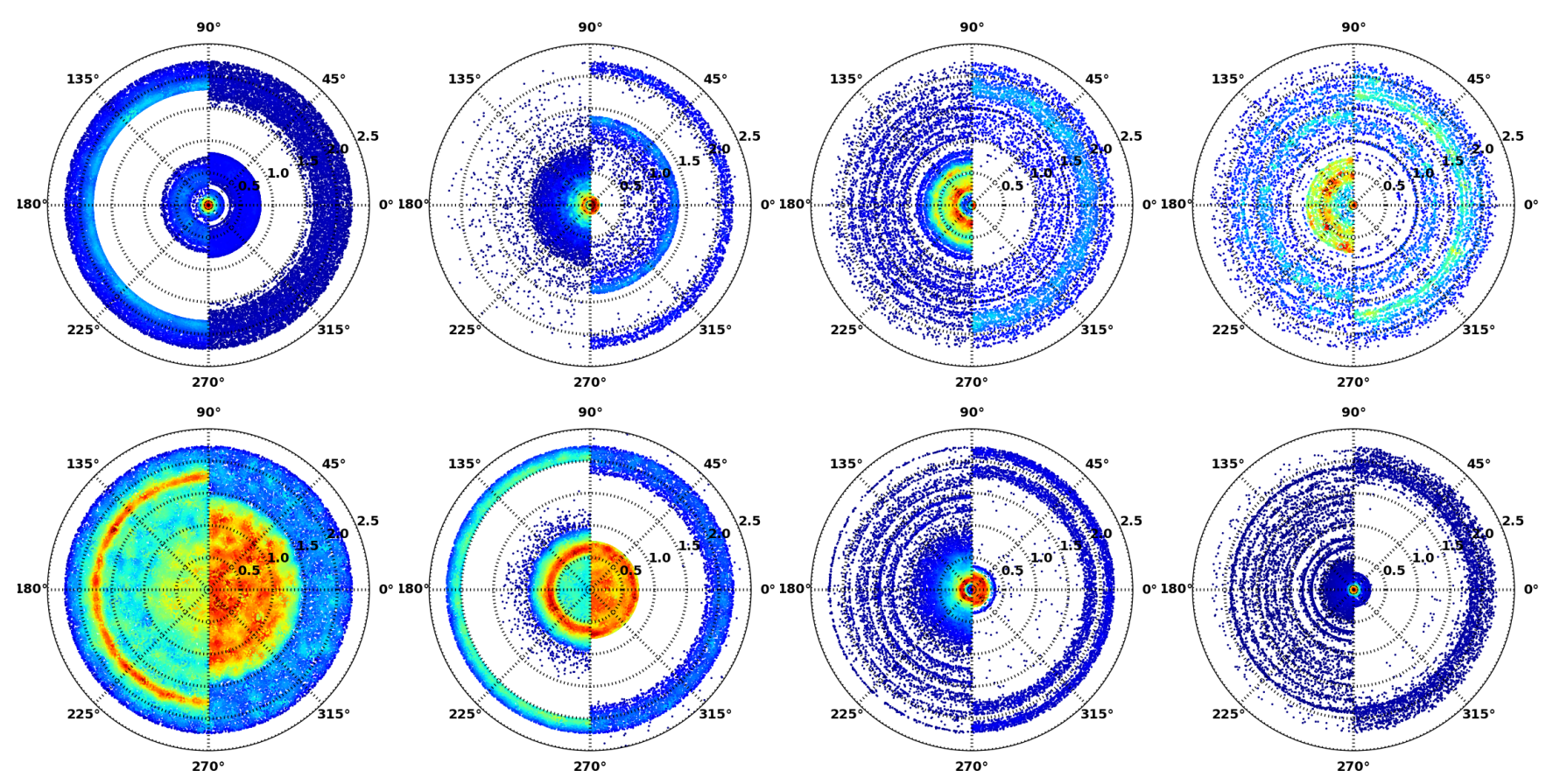

$90^{\circ}$

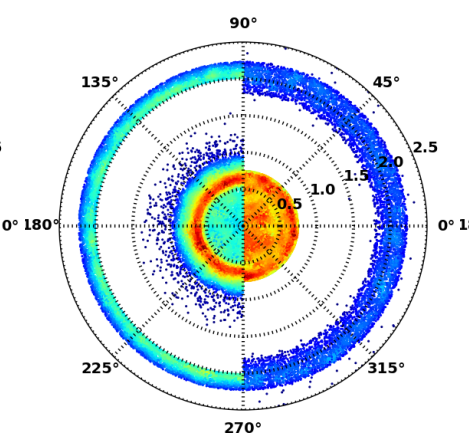

$90^{\circ}$

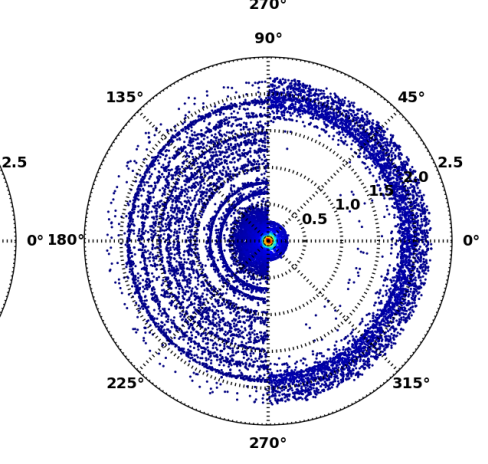

$90^{\circ}$

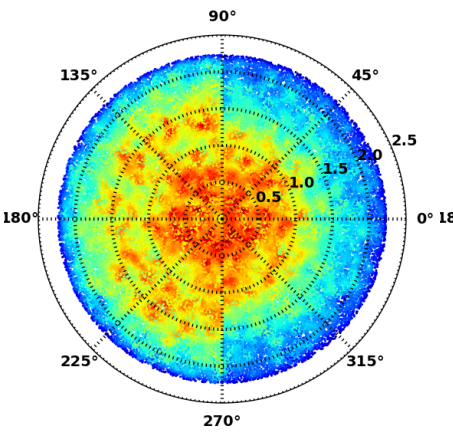

$90^{\circ}$

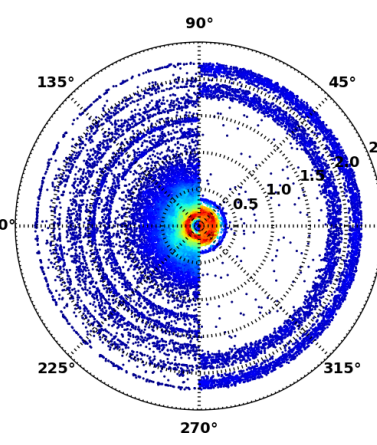

$90^{\circ}$
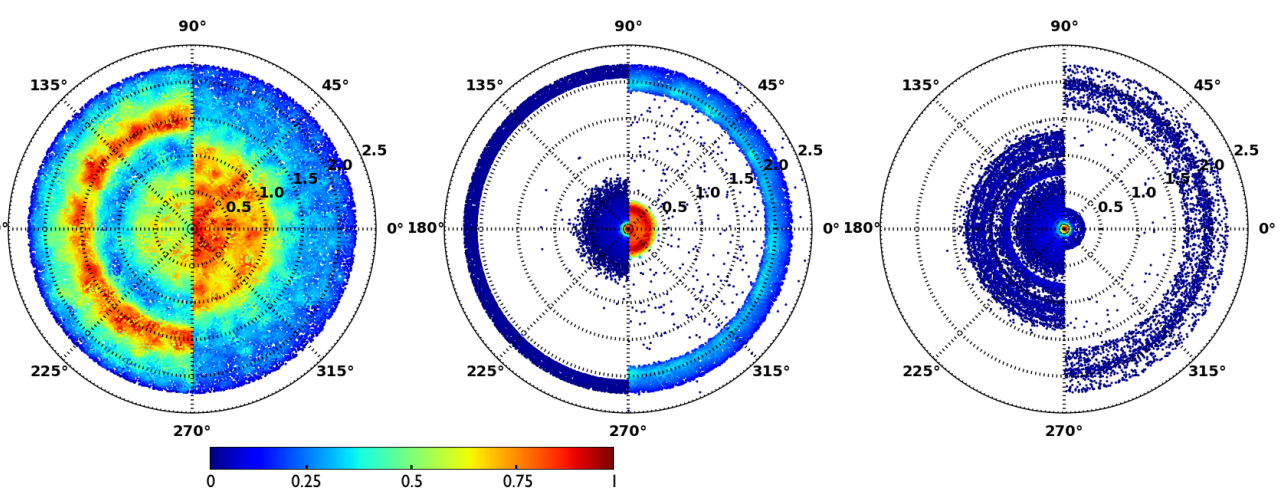

Figure 3. Escape location of charged particles along the CD of the BSPWN, in polar coordinates for the fully axisymmetric case $\theta_{\mathrm{i}}=0^{\circ}$. The polar angle $\theta$ in the range [0,2.5] rad is measured along the $\mathrm{CD}$ from the head of the bow-shock (the symmetry axis of the nebula). Given the symmetry of the problem, in the left and right side of each panel we plot escape positions for particles of different sign in the range of azimuthal angle $\phi$ either $[0, \pi]$ or $[\pi, 2 \pi]$. The color scheme ranges from 0 to 1 , and represents the relative density of points computed with a Gaussian kernel of FWHM $=0.02$ in units of the stand-off distance on the projection plane. Rows from top to bottom show cases A, B and C. Columns from left to right represent different effective rigidities $\mathcal{R}=1,0.5,0.25,0.1$.

- The fraction of escaping particles and the escape geometry is strongly energy dependent, and changing the energy from $\mathcal{R}=0.1$ to $\mathcal{R}=1$ can be enough to pass from an almost complete confinement, where most of the particles are advected in the tail, to an almost free escape. This means that energy independent arguments, in the description of how particles escape, are likely to be strongly inadequate, and only a detailed modeling of the full trajectories can give trustable results.

- Current sheets and lines are important confinement agents and they can strongly affect the pattern of escaping particles. This means that the structure, stability and dissipation of those features can substantially affect the way particles emerge out af these systems. In this respect the ability to properly model turbulence and dissipation, as key factors regulating those currents, becomes important for the level of anisotropy in the escape.

- Particle escape is likely to be charge separated (strong charge asymmetry) or partially charge separated. If the high energy particles that escape carry a sizable fraction of the overall energetics, this means that BSPWNe are likely to be characterized by the presence of charge separated flows. Charge separated flows, (and the related return currents) can give rise to filamentation, Weibel, and two-stream instabilities, which might affect deeply the underlying MHD structure that is based on charge neutrality.

One of the main issue related to the escape of high energy particle is that in systems like the Guitar nebula (Hui \& Becker 2007) or the Lighthouse nebula (Pavan et al.2014) one sided bright $\mathrm{X}$-ray feature are observed, which if interpreted in terms of high energy particles streaming out of the nebula into the ISM magnetic field (Bandiera 2008), require a large level of anisotropy. Our results show that the required anisotropy can be due to the internal magnetic field. Unlike reconnection, which only affects low energy particles with Larmor radii smaller that the extent of the reconnection site, which in general are much smaller than the size of the bow-shock, global magnetic effects are relevant also for $\mathcal{R} \sim 1$, and they generate large anisotropy over the entire bow-shock head.

Even of greater importance is the fact that the escaping parti- 

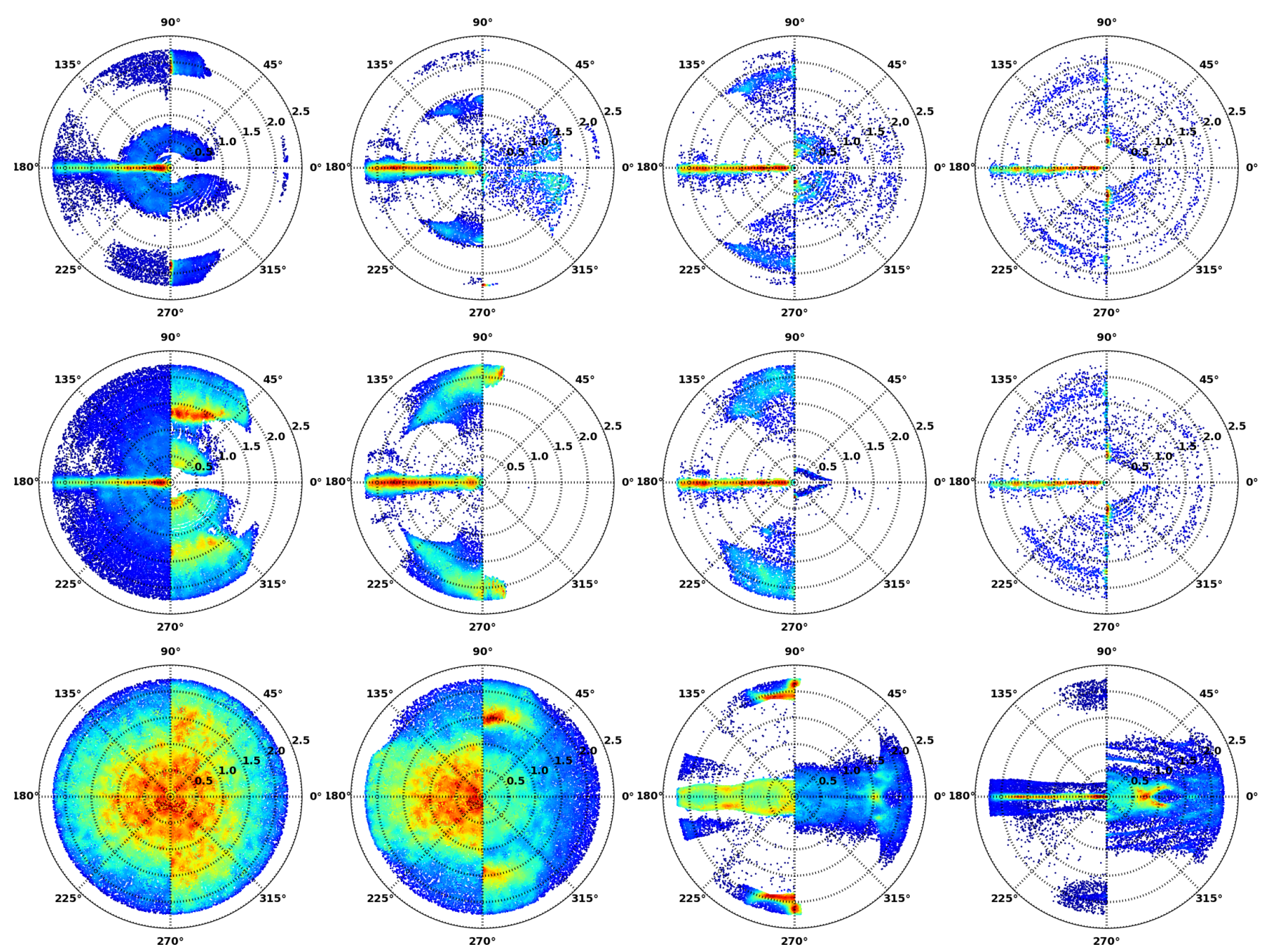

Figure 4. Same as Fig. 3 but for the orthogonal case $\theta_{\mathrm{i}}=90^{\circ}$.

cles might be in the form of a charge separated flow. This is highly relevant in terms of self confinement of the escaping particles. As the high energy particles start to stream in the ISM, they can drive streaming instability in a fashion not dissimilar to that of cosmic rays accelerated at SN shocks (Ptuskin, Zirakashvili \& Plesser 2008. Malkov et al. 2013, Nava et al. 2016, D'Angelo et al. 2018). The level at which induced turbulence saturate depends strongly on the level of charge separation. In the presence of a net current, the magnetic field can be amplified much more efficiently that for a neutral flow (Skilling 1971, Bell 2004). This means that it is far easier to self confine the escaping particles in the vicinity of the BSPWN, if the outflow is charge separated. Indeed the recent TeV halo detected around Geminga (Abeysekara et al.2017), which has been interpreted as an evidence for a larger ISM turbulence that what is commonly assumed, could instead be due to local magnetic field amplification by a charge separated pair outflow (Evoli, Linden \& Morlino 2018).

\section{ACKNOWLEDGEMENTS}

The authors acknowledged support from the PRIN-MIUR project prot. 2015L5EE2Y "Multi-scale simulations of high-energy astrophysical plasmas".

\section{REFERENCES}

Abeysekara A. U. et al., 2017, Science, 358, 911

Adriani O. et al., 2009, Nature, 458, 607

—, 2013, Soviet Journal of Experimental and Theoretical Physics Letters, 96, 621

Aguilar M. et al., 2013, Physical Review Letters, 110, 141102

Arzoumanian Z., Cordes J., Van Buren D., Corcoran M., SafiHarb S., Petre R., 2004, in Bulletin of the American Astronomical Society, Vol. 36, AAS/High Energy Astrophysics Division \#8, p. 951

Bandiera R., 2008, A\&A, 490, L3

Barkov M. V., Lyutikov M., 2018, ArXiv e-prints

Bell A. R., 2004, MNRAS, 353, 550

Bell J. F., Bailes M., Manchester R. N., Weisberg J. M., Lyne A. G., 1995, ApJLett, 440, L81

Blasi P., Amato E., 2011, Astrophysics and Space Science Proceedings, 21, 624

Bogovalov S. V., Chechetkin V. M., Koldoba A. V., Ustyugova G. V., 2005, MNRAS, 358, 705

Brownsberger S., Romani R. W., 2014, ApJ, 784, 154

Bucciantini N., 2002, A\&A, 387, 1066

-, 2018, MNRAS

Bucciantini N., Amato E., Del Zanna L., 2005, A\&A, 434, 189 

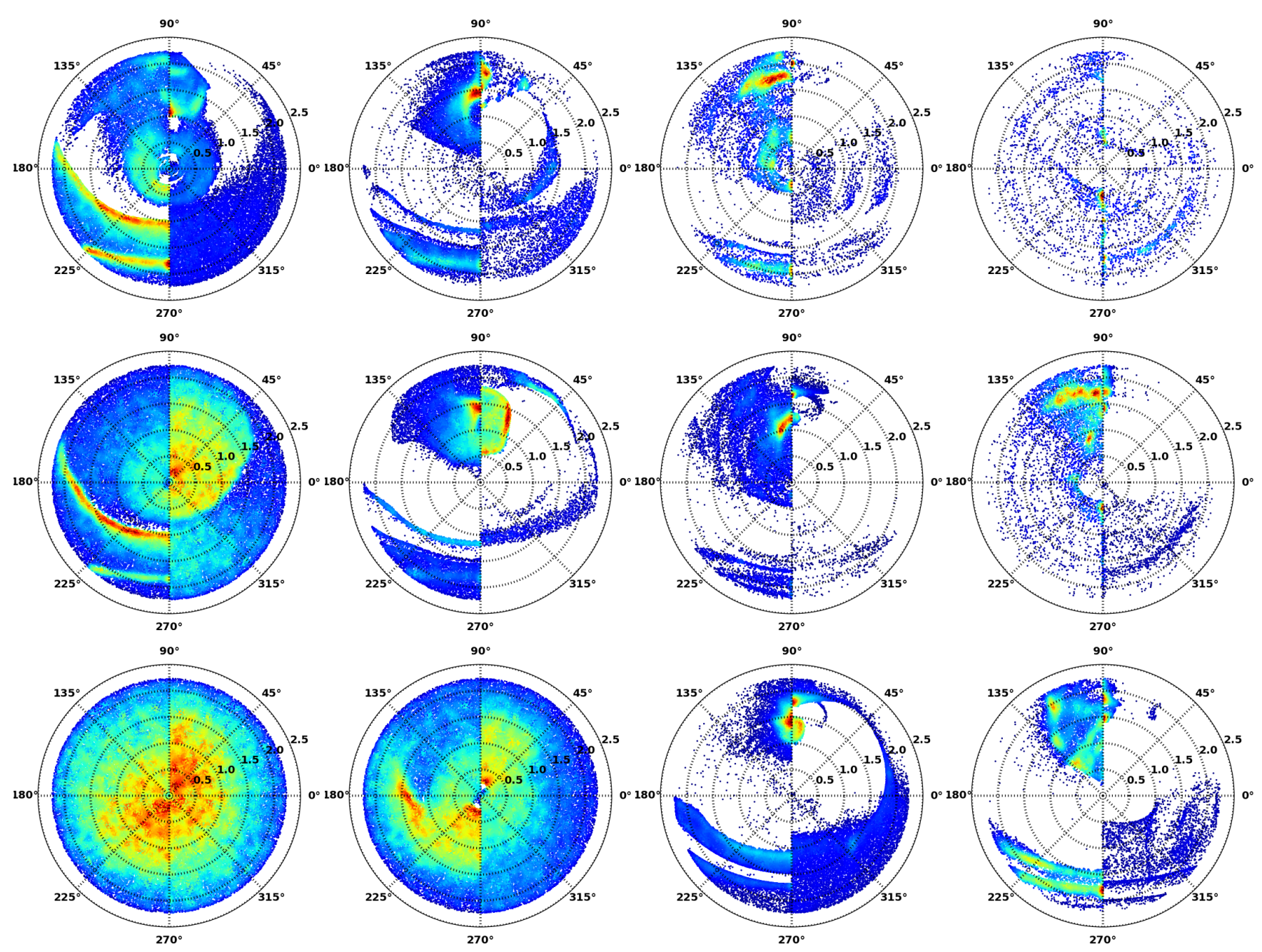

Figure 5. Same as Fig. 3 but for the inclined case $\theta_{\mathrm{i}}=45^{\circ}$.

Bucciantini N., Bandiera R., 2001, A\&A, 375, 1032

Chatterjee S., Gaensler B. M., Vigelius M., Cordes J. M., Arzoumanian Z., Stappers B., Ghavamian P., Melatos A., 2005, in Bulletin of the American Astronomical Society, Vol. 37, American Astronomical Society Meeting Abstracts, p. 1470

Contopoulos I., Kazanas D., Fendt C., 1999, ApJ, 511, 351

Cordes J. M., Romani R. W., Lundgren S. C., 1993, Nature, 362, 133

D’Angelo M., Morlino G., Amato E., Blasi P., 2018, MNRAS, 474

De Luca A. et al., 2011, ApJ, 733, 104

Del Zanna L., Amato E., Bucciantini N., 2004, A\&A, 421, 1063

Del Zanna L., Olmi B., 2017, in Astrophysics and Space Science Library, Vol. 446, Modelling Pulsar Wind Nebulae, Torres D. F., ed., p. 215

Evoli C., Linden T., Morlino G., 2018, ArXiv e-prints

Faganello M., Califano F., Pegoraro F., Andreussi T., Benkadda S., 2012, Plasma Physics and Controlled Fusion, 54, 124037

Fear R. C., Trenchi L., Coxon J. C., Milan S. E., 2017, Journal of Geophysical Research (Space Physics), 122, 12

Frey H. U., Phan T. D., Fuselier S. A., Mende S. B., 2003, Nature, 426, 533

Fuselier S. A., Frahm R., Lewis W. S., Masters A., Mukherjee
J., Petrinec S. M., Sillanpaa I. J., 2014, Journal of Geophysical Research (Space Physics), 119, 2563

Fuselier S. A., Trattner K. J., Petrinec S. M., Lavraud B., 2012, Journal of Geophysical Research (Space Physics), 117, A08235

Gaensler B. M., 2005, Advances in Space Research, 35, 1116

Gaensler B. M., Slane P. O., 2006, ARA\&A, 44, 17

Gaensler B. M., van der Swaluw E., Camilo F., Kaspi V. M., Baganoff F. K., Yusef-Zadeh F., Manchester R. N., 2004, ApJ, 616,383

Gallant Y. A., 2007, Ap\&SS, 309, 197

H. E. S. S. Collaboration et al., 2018, A\&A, 612, A2

-, 2014, A\&A, 562, A40

Hales C. A., Gaensler B. M., Chatterjee S., van der Swaluw E., Camilo F., 2009, ApJ, 706, 1316

Helfand D. J., Gotthelf E. V., Halpern J. P., Camilo F., Semler D. R., Becker R. H., White R. L., 2007, ApJ, 665, 1297

Hooper D., Blasi P., Dario Serpico P., 2009, JCAP, 1, 025

Hui C. Y., Becker W., 2007, A\&A, 467, 1209

Jakobsen S. J., Tomsick J. A., Watson D., Gotthelf E. V., Kaspi V. M., 2014, ApJ, 787, 129

Jones D. H., Stappers B. W., Gaensler B. M., 2002, A\&A, 389, L1

Kan J. R., 1988, J. Geophys. Res., 93, 5613

Kargaltsev O., Misanovic Z., Pavlov G. G., Wong J. A., Garmire 
G. P., 2008, ApJ, 684, 542

Kargaltsev O., Pavlov G. G., Klingler N., Rangelov B., 2017, Journal of Plasma Physics, 83, 635830501

Kirk J. G., Skjæraasen O., 2003, ApJ, 591, 366

Klingler N. et al., 2016, ApJ, 833, 253

Komissarov S. S., Lyubarsky Y. E., 2004, MNRAS, 349, 779

Kulkarni S. R., Hester J. J., 1988, Nature, 335, 801

Li X. H., Lu F. J., Li T. P., 2005, ApJ, 628, 931

Lyubarsky Y., Kirk J. G., 2001, ApJ, 547, 437

Lyubarsky Y. E., 2003, MNRAS, 345, 153

Malkov M. A., Diamond P. H., Sagdeev R. Z., Aharonian F. A.,

Moskalenko I. V., 2013, ApJ, 768, 73

Marelli M. et al., 2013, ApJ, 765, 36

Misanovic Z., Pavlov G. G., Garmire G. P., 2008, ApJ, 685, 1129

Nava L., Gabici S., Marcowith A., Morlino G., Ptuskin V. S., 2016, MNRAS, 461, 3552

Ng C.-Y., Bucciantini N., Gaensler B. M., Camilo F., Chatterjee S., Bouchard A., 2012, ApJ, 746, 105

$\mathrm{Ng} \mathrm{C}$. Y. et al., 2009, in Bulletin of the American Astronomical Society, Vol. 41, American Astronomical Society Meeting Abstracts \#213, p. 307

Ng C.-Y., Gaensler B. M., Chatterjee S., Johnston S., 2010, ApJ, 712,596

Olmi B., Del Zanna L., Amato E., Bucciantini N., Mignone A., 2016, Journal of Plasma Physics, 82, 635820601

Pavan L. et al., 2014, A\&A, 562, A122

Pinnock M., Rodger A. S., Dudeney J. R., Rich F., Baker K. B., 1995, Annales Geophysicae, 13, 919

Porth O., Komissarov S. S., Keppens R., 2014, MNRAS, 438, 278

Posselt B. et al., 2017, ApJ, 835, 66

Ptuskin V. S., Zirakashvili V. N., Plesser A. A., 2008, Advances in Space Research, 42, 486

Romani R. W., Slane P., Green A. W., 2017, ApJ, 851, 61

Scholer M., 2003, in Lecture Notes in Physics, Berlin Springer Verlag, Vol. 612, Energy Conversion and Particle Acceleration in the Solar Corona, Klein L., ed., pp. 9-27

Sironi L., Spitkovsky A., 2009, ApJ, 698, 1523

—, 2011, ApJ, 741, 39

Skilling J., 1971, ApJ, 170, 265

Spitkovsky A., 2006, ApJLett, 648, L51

—, 2008, ApJLett, 682, L5

Tchekhovskoy A., Philippov A., Spitkovsky A., 2016, MNRAS, 457, 3384

Timokhin A. N., 2006, MNRAS, 368, 1055

van Kerkwijk M. H., Kulkarni S. R., 2001, A\&A, 380, 221

Vigelius M., Melatos A., Chatterjee S., Gaensler B. M., Ghavamian P., 2007, MNRAS, 374, 793

Volpi D., Del Zanna L., Amato E., Bucciantini N., 2008, in American Institute of Physics Conference Series, Vol. 983, 40 Years of Pulsars: Millisecond Pulsars, Magnetars and More, C. Bassa, Z. Wang, A. Cumming, \& V. M. Kaspi, ed., pp. 216-218

Wang W., Pun C. S. J., Cheng K. S., 2006, A\&A, 446, 943

Wilkin F. P., 1996, ApJLett, 459, L31

Yusef-Zadeh F., Gaensler B. M., 2005, Advances in Space Research, 35, 1129 